\title{
TELÉFONOS MÓVILES, COMUNICACIÓN E INTERACCIÓN
}

\section{Mobile phones, communication and interaction}

Amaro La Rosa*

\begin{abstract}
Resumen
El presente artículo intenta una aproximación a las modalidades de interacción que se hacen patentes en el uso de los teléfonos móviles, analizando su vinculación con la interacción cara a cara y describiendo sus particularidades en un mundo caracterizado por la movilidad y la convergencia de las comunicaciones.
\end{abstract}

Palabras clave: Teléfonos móviles, Interacción, Comunicación.

\begin{abstract}
This article attempts an approach to the modalities of interaction in the use of mobile phones, analyzing their relationship with the face to face interaction and describing its particularities in a world characterized by mobility and convergence of communications.
\end{abstract}

Key words: Mobile phones, Interaction, Communication

* Docente de la Universidad Femenina del Sagrado Corazón. amaro@unife.edu.pe 
Décadas atrás cuando una persona que vivía en cualquier ciudad salía rumbo a su trabajo, quedaba un margen de tiempo que podríamos llamar de latencia durante el cual si era necesario que se enterara de algo urgente que sucedía en casa había que medir el tiempo que demoraba en el trayecto para llamarle luego, y si se trataba de algo muy privado debía esperarse hasta su retorno que eventualmente era 12 horas después. Antes se rompía la conexión con la familia momentáneamente y resultaba normal que así pasara pues había una distancia muy marcada entre su vida familiar privada y el mundo público del trabajo. Ahora en muchos casos, apenas la persona sale de casa ya está recibiendo alguna llamada o SMS de los suyos, o es en todo caso la misma persona quien telefonea para averiguar cómo resultó algo que dejó inconcluso hace solamente unos minutos. En especial, es muy frecuente que los jóvenes quienes acaban de despedirse en la puerta de su escuela sigan conectados momentos después a través de mensajes de texto o llamadas telefónicas y que continúen haciéndolo cuando lleguen a sus hogares. Es este el mundo posmoderno en que la interacción cara a cara se produce cuando es posible y en caso contrario se reemplaza por la comunicación móvil que tiene la ventaja de conectar a los individuos que se encuentran en espacios distintos, pero que comparten el mismo espacio virtual.

¿Cuánto ha cambiado la vida cotidiana de las personas desde que los teléfonos móviles al ser parte de la economía de escala se convirtieron en productos asequibles a la mayoría?

\section{MÓVILES HOY}

La telefonía móvil se ha desarrollado aceleradamente en los últimos años, y la demanda por el servicio crece en la medida en que la población ya no lo percibe como un lujo sino como una necesidad para estar comunicado constantemente.

En la actualidad, en muchas ciudades se encuentra funcionando un número sensiblemente mayor de teléfonos celulares que de teléfonos fijos. Ello se explica entre otras razones, porque sus menores costos en cuanto al servicio y al propio aparato los hacen asequibles para los diversos sectores sociales. Asimismo, porque las exigencias laborales o sociales demandan que algunas personas deban utilizar varios teléfonos. Según el Banco Mundial (2011) existen diversos países que tienen más teléfonos móviles que habitantes. Los líderes en penetración de celulares aparecen en el cuadro 1

\section{CUADRO 1 \\ PAÍSES CON MAYOR PENETRACIÓN DE CELULARES}

\begin{tabular}{lc}
\hline PAÍ́S & $\begin{array}{r}\text { TASA DE PENETRACIÓN } \\
\text { (\% por } 100 \text { habitantes) }\end{array}$ \\
\hline Emiratos Árabes Unidos & 232 \\
Estonia & 203 \\
Bahrein & 199 \\
Macao' & 193 \\
Arabia Saudita & 177 \\
Qatar & 175 \\
Hong Kong' & 174 \\
Saint Kits y Nevis & 167 \\
Panamá & 165 \\
Rusia & 164 \\
\hline
\end{tabular}

'Región Autónoma Especial, República Popular China Fuente: The World Bank (2011)

Actualmente es común escuchar, antes de iniciarse una conferencia, un concierto o una función de teatro, la recomendación de apagar previamente los celulares para evitar interrupciones, ante lo cual aquellos partidarios del "derecho humano de mantenerse siempre conectados" optan por el uso del vibrador. En los exámenes de admisión a las universidades se prohíben expresamente, pues en diversas ocasiones se ha sorprendido a postulantes recibiendo respuestas a través del teléfono móvil. Del mismo modo, en muchas instituciones se exige a los visitantes dejar su aparato en la oficina de recepción, por motivos de seguridad; tal como en el caso de los bancos y otras entidades financieras. Esta medida se adoptó luego de detectarse que los delincuentes empleaban estos equipos para coordinar sus robos. Asimismo, antes de decolar los aviones comerciales, se avisa expresamente a los propietarios de teléfonos móviles que suspendan su uso para evitar que se produzcan interferencias con los equipos electrónicos de a bordo. 
En la era de la convergencia de las telecomunicaciones ya no podemos referirnos a Internet como algo alejado de la telefonía móvil. Por el contrario, cada vez más los operadores locales se interesan porque sus clientes adquieran equipos $4 \mathrm{G}$ (con acceso inalámbrico de alta velocidad) y que empleen sus móviles para navegar por el ciberespacio. De ello da fe la diversidad de ofertas que pueden observarse actualmente en el mercado global. En muchos casos suponen entregar un equipo a menor precio que el ofertado en plaza, pero firmando un contrato no menor de un año para hacer uso de Internet. Obviamente lo que aquí interesa es el uso del servicio, en la práctica algo mayor que el vigente en un servicio de línea dedicada a través de un teléfono fijo. Para Martínez (2008), la ciudad es el lugar por excelencia del hombre posmoderno y es precisamente en las metrópolis en donde se siente la omnipresencia de la comunicación digital, especialmente de las redes sociales y los teléfonos móviles que paulatinamente se convierten en unos de sus vehículos más importantes. No obstante, no puede dejar de considerarse su creciente impacto en zonas rurales. Una reciente investigación desarrollada en Papua Nueva Guinea, comprueba que las dos principales ventajas de los móviles en zonas rurales ( $88 \%$ del país), son para la satisfacción de necesidades de comunicación y para la interacción social con los seres queridos (Watson, 2011, p. iv)

\section{TELÉFONO MÓVIL, VIDA COTIDIANA Y COMUNICACIÓN}

En realidad, los celulares se han convertido en parte de la vida cotidiana de millones de personas en el mundo entero. Esto es, recogiendo las palabras de Prieto (1994) la tecnología está integrada como un objeto privilegiado en el contexto de interacción simbólica y conductual en el cual se desenvuelve el ser humano. La percepción que tiene el usuario del dispositivo, acerca de su equipo móvil, es la un instrumento interactivo que resuelve sus problemas de comunicación y de otros más que dependen de su configuración. Y es precisamente esa evidencia la que eventualmente produce una marcada dependencia en el individuo usuario.

A nuestro entender el uso de los teléfonos móviles ha causado un impacto considerable en la comunicación. Consideramos que ésta puede hacerse evidente en:

1. Formas preexistentes de comunicación: La comunicación telefónica ya existía: no obstante los móviles aportan la posibilidad de comunicarse desde y hacia casi cualquier lugar, estén o no los individuos movilizándose de un lugar a otro.

2. Nuevas modalidades de comunicación: La telefonía móvil a partir de la segunda generación $(2 \mathrm{G})$ introduce los mensajes de texto (SMS) como recurso para incrementar las posibilidades de comunicación, la cual es especialmente empleada de manera permanente por los usuarios jóvenes

Para Martínez y Aguado (2006) el desarrollo de la telefonía móvil supone una segunda revolución digital caracterizada por:

1. Translocalidad: Comunicación en trayectorias de desplazamiento; esto es, independiente del lugar,

2. Integración: Coordinación de aplicaciones, funciones y servicios en un metadispositivo digital de comunicación y acceso.

Por su parte, Palen (2001) señala que la conectividad que producen los teléfonos móviles no se reduce a lo tecnológico sino a la conexión social de múltiples aspectos de la vida de los individuos a los cuales la autora describe con casos concretos como el cruce del espacio y tiempo, el incremento de la accesibilidad y la expansión de las redes sociales (Gráfico 1). 


\section{GRÁFICO 1}

\section{CONECTIVIDAD DE LOS TELÉFONOS MÓVILES}

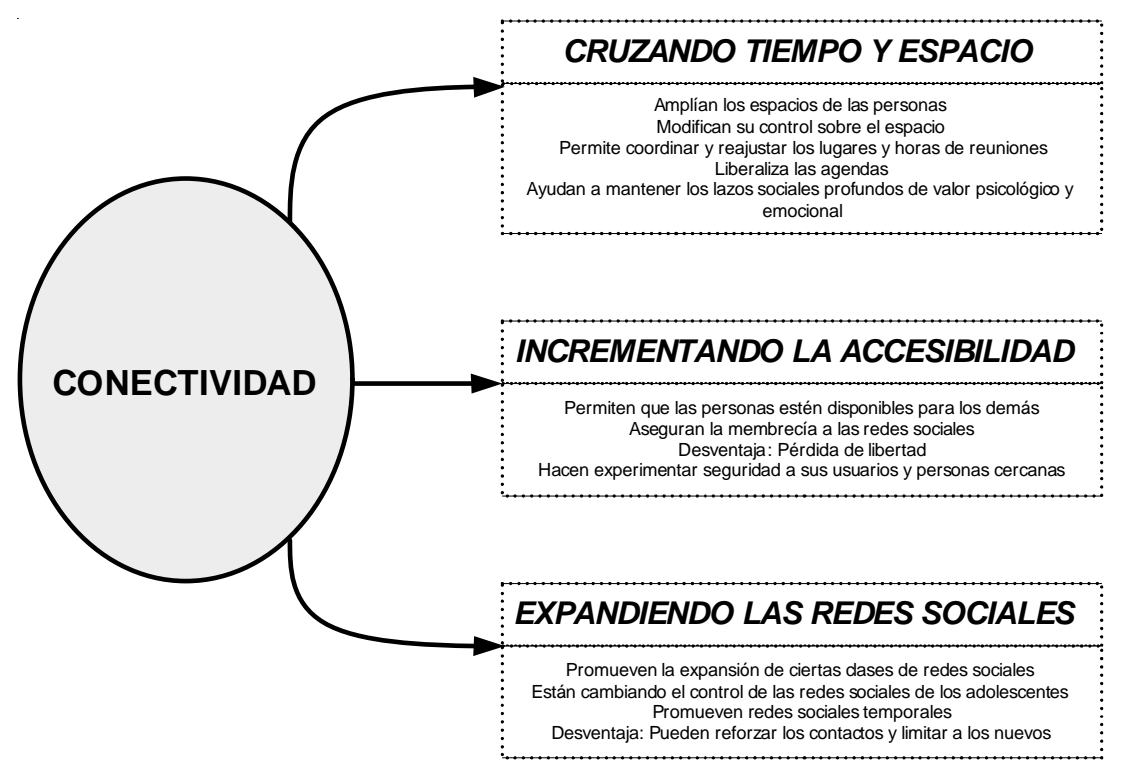

Basado en Palen (2001). Elaboración: ALR

\section{INTERACCIÓN EN EL ESPACIO MÓVIL}

Cuando dos sujetos interactúan se establece entre ellos un circuito de comunicación, el cual obviamente no es un sistema cerrado como el de un circuito eléctrico. Configuran un sistema abierto, cambiante, dado que son seres humanos quienes interactúan y quienes no permanecen comunicándose exclusivamente entre sí, puesto que entablan a su vez comunicación con otras personas de su red social, sea de manera directa en una comunicación cara a cara o usando un dispositivo tal como el teléfono móvil, en cuyo caso por cierto los patrones de interacción han experimentado un cambio profundo (Geser, 2004).

Tal como ya se ha comprobado de manera fehaciente, la comunicación interpersonal está tan íntimamente ligada con la interacción que la existencia de una presupone necesariamente la otra. Haciendo un ejercicio de abstracción intentaremos explicar cómo se relacionan entre sí en el intercambio comunicativo móvil, que tal como observaremos significa mucho más que hablar utilizando este dispositivo.
El ser humano en la medida que desarrolla modalidades de comportamiento social concordantes con las expectativas de su entorno, las cuales poseen evidentes patrones normativos, interioriza igualmente el manejo de los códigos empleados por los demás en su comunicación cotidiana. Es en este entorno de interacción-comunicación, que los teléfonos móviles son actualmente un recurso muy utilizado por los individuos para interrelacionarse. De acuerdo al segmento social en que nos encontremos y del estilo de vida que poseamos, vamos a otorgarle mayor o menor importancia al teléfono móvil. Es por ello que algunos especialistas lo catalogan como un factor que debilita la interacción.

Rettie (2009) sostiene que es posible aplicar los criterios sobre la interacción planteados por Goffman a la comunicación vía teléfono móvil y en general a la comunicación mediada sincrónica. Coincidiendo con este criterio, Ling (2008, p. 119), afirma que los móviles pueden emplearse para facilitar e incrementar la interacción a pequeña escala y que en el entorno de esta interacción mediatizada se hacen patentes diversas modalidades de rituales: 
1. Saludos pre-configurados.

2. Negociación de relaciones románticas.

3. Mensajes de texto y definición de los límites del grupo

4. Bromas

5. Réplicas agudas

6. Chismes

Por su parte, desde una visión construccionista, la cual supone que los objetos o hechos de la realidad son construidos en el marco de la actividad social humana, Gergen (2002) se refiere a la presencia ausente, que constituye la presencia física, mental y social en varios lugares a la vez, que se hace evidente con la utilización de los teléfonos móviles. El gráfico 2 visualiza la manera en que se produce una situación de interacción cotidiana, donde el individuo se mantiene en contacto con alguien a través de su móvil mientras que permanece en un espacio físico donde está presente una tercera persona.

\section{GRÁFICO 2 \\ LA PRESENCIA AUSENTE}

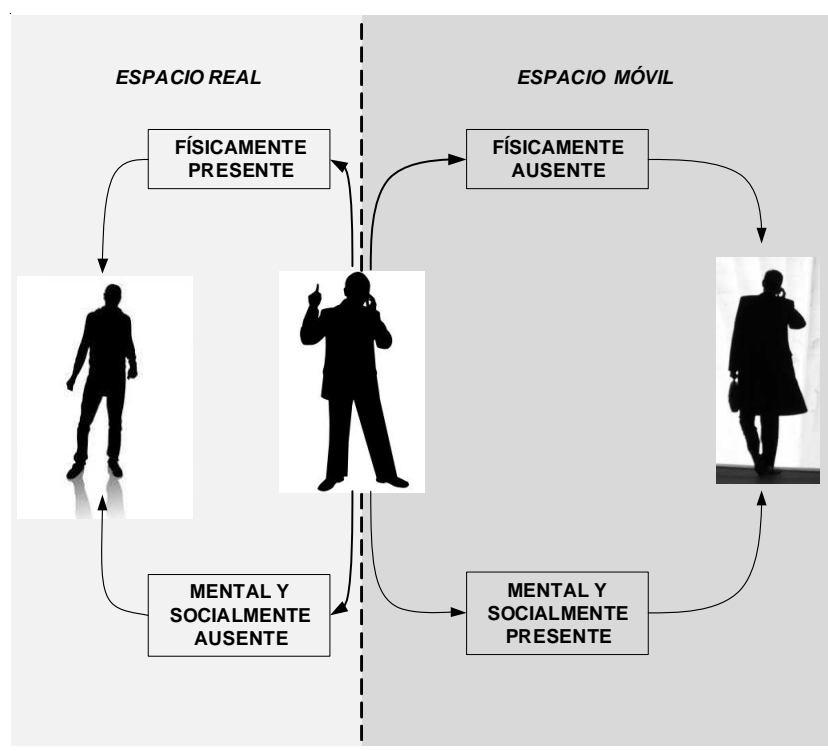

Basado en Gergen (2002). Elaboración: ALR

Para lograr una comunicación más fluida y una interacción apropiada se requiere contar con las competencias necesarias para utilizar cabalmente todos los recursos de los instrumentos que posibilitan el intercambio. Así podemos notar cómo los jóvenes manejan con suma rapidez y destreza sus dedos pulgares para componer los textos y números en un mensaje cualquiera, mientras que con la misma mano sostienen su teléfono móvil.

Los recursos que actualmente posee un teléfono "inteligente" son tan variados que todos los usuarios no los conocen a plenitud. Probablemente los nativos digitales hagan uso de un mayor número de funciones que los migrantes digitales, pero aún así existen diferencias entre los integrantes de estas categorías, que dependen de sus competencia individuales.

A decir de Oksman \& Turtiainen (2004) los canales de comunicación multimedia establecidos por los adolescentes mediante el uso de sus teléfonos móviles les sirven para articular sus espacios personales, posibilitar su presentación del yo y definir sus relaciones con los demás. Sobre el particular Seguí (2009) afirma que los roles que desempeñan los nativos digitales en la interacción constituyen una forma de co-presencia en un universo que ellos mismos construyen en conjunto.

Caronia \& Caron (2004) describen la manera en que los teléfonos móviles pasan a formar parte de la vida diaria de los adolescentes convirtiéndose en una suerte de detonadores del pensamiento social. A través de su uso los adolescentes construyen su identidad y aprenden a presentar su yo en la esfera pública. El uso de los móviles demanda el desarrollo de las propias competencias que los habilitan como individuos funcionales a su sociedad.

De esta manera, los teléfonos móviles se convierten en importantes recursos para la socialización. Al ser el uso de móviles un elemento facilitador de relaciones interpersonales, se relaciona de manera directa con la necesidad de pertenencia y la identidad social que son constructos importantes en el proceso de socialización (Walsh, 2009) Sobre el particular, Cassidy (2006) encuentra que los adolescentes que intentan se vistos de manera más positiva por sus pares, se sienten más presionados a usar sus teléfonos móviles. A nuestro entender, este criterio ha sido recientemente validado por la investigación de Walsh, White, Cox \& Young (2011) quienes encuentran que 
la autoestima influye en la necesidad de pertenencia y que ésta, en relación con las normas intragrupales, se asocia con la autoidentificación de los jóvenes australianos de 16 a 24 años como usuarios de teléfonos móviles.

Es muy probable en estos casos, también se produzca una rápida obsolescencia de la tecnología que utilizan; así al momento de redactar este artículo, los Blackberrys se hacían cada vez más populares entre los jóvenes de Lima, lo cual era reforzado por cierto con estrategias de comercialización de las empresas operadoras que ofrecían, en especial a estudiantes universitarios, equipos a un costo considerablemente bajo.

Cuando entre dos sujetos se desarrolla una relación determinada se configura una estructura funcional que depende del modo en que se comuniquen entre sí. Desde la perspectiva de Humphreys (2010) la proliferación de los teléfonos móviles y de las redes sociales que ahora vehiculizan, están transformando considerablemente la manera en que las personas se reúnen e interactúan. Así, cuando padres e hijos se encuentran en el hogar los móviles no son el centro de atención, pero cuando salen, sus aparatos se convierten en verdaderos articuladores del hogar, elementos que garantizan la presencia de los hijos, enlazados con la base hogareña donde funcionan los móviles de los padres (Palen y Hughes, 2007).

Lasén (2006) plantea la especificidad de la comunicación desarrollada con la intermediación de los móviles, la cual supone el mantenimiento de interacciones simultáneas virtuales así como cara a cara en el plano verbal o no verbal; pero además la posibilidad de realizar al mismo tiempo una serie de actividades de diversa índole.

Rheingold (2004) señala que los celulares establecen nuevos límites de identidad y lugar así como nuevas prácticas tecnosociales en las cuales se unen los estándares técnicos y las normas sociales. Los celulares se han interiorizado en las complejas tramas sociales desarrollando, gracias a los avances tecnológicos formas particulares de intercambio simbólico. Cabe aquí mencionar el estudio de Andrade (2008), quien encuentra que los SMS están generando en los jóvenes de la ciudad de Quito formas alternativas de relacionamiento, la popularización de varias maneras de construcción del lenguaje y consumos culturales que van más allá del sólo uso del artefacto.

Es evidente que ahora, especialmente entre los jóvenes y de manera creciente en los adultos, se acuerda cierto punto de reunión que es en todo caso un lugar probable, puesto que con frecuencia lo modifican e inclusive cambian la propia hora de reunión mediante empleando para este propósito mensajes de diverso tipo (SMS, voz, texto, etc.) convirtiéndose en mecanismos de coordinación y ajuste (Grinter \& Eldridge, 2001). Coincidiendo con este criterio, Gadarev (2006) opina que la movilidad de la cultura juvenil se hace evidente en todas las esferas. Ello produce un estilo flexible especial de la interacción comunicativa, que se expresa en una constante coordinación, cuando todas las decisiones se toman "sobre la marcha" y se pueden cambiar en cualquier momento. Por cierto podríamos asumir que esta movilidad es una característica de la sociedad actual en su conjunto.

En el plano interpersonal es imposible interactuar sin comunicarse, tal como lo han planteado reiteradamente los teóricos de la Escuela de Palo Alto. Cuando dos personas se conectan virtualmente a través de los móviles, evidentemente se comunican, cualquiera sea el propósito por el cual la establezcan. Ello al margen de que puedan ser diferentes los propósitos del intercambio, su naturaleza, o el comportamiento social de los implicados, lo cual decide la especificidad de la comunicación

En los años 70 los niños latinoamericanos eran motivados para acostarse contando con el mensaje del Topo Gigio, el ícono de moda de entonces, que reclamaba tiernamente a su partenaire en las pantallas de TV "el besito de las buenas noches" para irse a dormir. El estudio de Grinter y Eldridge (2001) comprueba que, aprovechando la intimidad del espacio privado que permite el teléfono móvil y la silenciosa complicidad del mensaje de texto, enamorados y amigos cercanos intercambian saludos de despedida en la noche. Y realmente, muchos adolescentes no concilian el sueño mientras no reciban su virtual "besito de las buenas noches". Realizando un ejercicio mental de conversión de la ficción en realidad, Romeo y Julieta 
de la era digital expresarían así su afecto de manera clandestina, sin que siquiera se enteraran los Montesco ni los Capuleto; por más vigilantes que se mantuvieran.

Evidentemente en la comunicación interpersonal se emplean diversos canales para comunicar. No obstante existen indicadores de multicanales en la comunicación a través de los móviles. Langer, Moyer y Stoner (2009) señalan que los adolescentes prefieren el uso de mensajes de texto porque les facilita el uso de múltiples canales de conversación que actúan simultáneamente, inclusive mientras están desarrollando sus tareas en la computadora. Tanto más, los SMS tienen un significado per se que es el de demostrar al destinatario la importancia de su contacto. Para los jóvenes involucra una consolidación de sus relaciones sociales, Las mismas que se configuran en el entorno de las redes sociales con las que interactúan en su vida cotidiana, las cuales aportan al individuo sociabilidad, apoyo, información así como sensación de pertenencia e identidad (Andrade, 2008, pp. 18-19).

El estudio de Faulkner y Culwin (2005) demuestra que son las mujeres quienes envían un mayor número de mensajes de texto, demostrando un mayor grado de sensibilidad en los intercambios, que se grafica, en la mayor frecuencia de uso de emoticones.

Desde el punto de vista de Ling (2008) al interactuar con los móviles pueden hacerse efectivas dos modalidades:

- Interacción verbal en la comunicación oral establecida.

- Interacción a través de mensajes de texto

Algo que estimamos no ha sido investigado suficientemente, tal vez por la complejidad o la fugacidad de sus manifestaciones es el uso de los recursos de comunicación no verbales ligado con el empleo de celulares. En la era de la comunicación y contando con sofisticados recursos para el registro de imágenes, estimamos que es un terreno que podrían abordar los especialistas.

En contra de lo simple que pudiera parecer, la problemática del uso de los teléfonos móviles es un asunto de marcada complejidad. A decir de van Biljon
\& Krotzké (2008) involucra al aparato telefónico, el sistema de telecomunicaciones con el cual está conectado, los usuarios de móviles, la adopción de éstos y el propio uso del sistema de teléfonos móviles. Cada uno de estos elementos tiene desde luego su propia naturaleza

Nuestro libro "La gente y su aparato: Historias de vida sobre teléfonos celulares"(La Rosa, 2008) revela a través de estudios de casos como estos dispositivos intervienen en el mundo cotidiano de las personas. Esta investigación nos ha permitido formular de manera preliminar un criterio sobre las funciones que cumplen los celulares, el cual hemos ampliado posteriormente, a partir de la presentación del modelo en diversas actividades científicas:

- Como instrumento de comunicación personal: Que es en principio lo que se debería esperar de un teléfono, pues para ello fue inicialmente concebido.

- Como recurso para una interacción más constante: Es factible comunicarse constantemente y ubicar a la persona que se desee dondequiera se encuentre el usuario. Esto es obviamente posible, dentro del rango de cobertura del servicio telefónico que ha contratado.

- Como sistema de ayuda: Se están empleando teléfonos móviles como instrumentos para discapacitados, ancianos y enfermos con Alzheimer. También se están utilizando aplicaciones para encontrar la ruta más descongestionada, los restaurantes y hasta para llegar rápidamente las estaciones de servicio más cercanas, cuando la gasolina está por agotarse.

- Como recurso lúdico: Los primeros juegos para celulares eran poco menos que el recordado Pac Man de los años 80. Ahora existe toda una industria que provee decenas de juegos en $3 \mathrm{D}$ y con alta definición, algunos incluidos como parte estándar del equipo y otros que pueden bajarse de la red como shareware, freeware o previo pago.. 
- Como elemento de entretenimiento: Escuchar música, ver dibujos animados y hasta telenovelas, ya no resulta una novedad en los celulares. A modo de ejemplo, en febrero del 2005, durante la tercera temporada de la exitosa serie 24 trasmitida por la cadena Fox, ya era factible encontrar una versión de la misma para teléfonos móviles (24: Conspiracy). En Japón las novelas escritas por celulares han tenido considerable éxito, siendo su best seller Cielo de amor, que inclusive ha sido llevada a la televisión en una miniserie dramática.

- Como medio testimonial: Con la tecnología digital pueden captarse, almacenarse y enviarse fotografías y videos en tiempo real, inmediatamente de ser captados ya sea hacia otro celular o hacia un correo electrónico. Así algunas de las primeras imágenes de los atentados en el metro de Londres fueron enviadas desde los móviles de personas que se encontraban en el lugar de los hechos.

- Como signo de estatus: En la actualidad, dada la considerable disminución de precios de los equipos y de las tarifas de telecomunicaciones, hasta los sectores de bajos ingresos pueden contar con un celular, pero no tendrán acceso (por el momento) a la generación más avanzada de equipos que tienen un costo de al menos US $\$ 500$ en el mercado local. Es por ello posible encontrar como usuarios de estos equipos a recicladores de basura y a vendedores ambulantes

- Como elemento de control: Millones de padres de familia en el mundo entero, emplean los celulares para seguir de cerca las actividades de sus hijos y naturalmente como recursos para su propia seguridad. Para ello, es factible encontrar en el mercado equipos con el Sistema de Posicionamiento Global (GPS). Por cierto que en el extremo negativo se encontraría la persona obsesiva con celos enfermizos (celotípico), para quien el equipo seria un recurso para someter a su pareja y monitorear sus actividades durante todo el tiempo posible.

- Como instrumento de negocios: Muchas compañías dotan con teléfonos móviles a sus empleados a fin de que puedan cumplir con sus funciones. Sin embargo, cabe anotar que desde hace años, los teléfonos móviles son utilizados en Lima con propósitos económicos por los cambistas $^{1}$, para conocer el valor actualizado de la moneda extranjera. Igualmente son utilizados por los agricultores de muchos países para conocer los precios de sus productos en los mercados de las ciudades, lo que implica un importante aporte para el desarrollo.

Desde luego, para miles de personas el dispositivo les sirve para realizar sus transacciones comerciales o mantener sus contactos de negocios.

\section{- Como instrumento de movilización social:} Para promover comportamientos colectivos de reacción social, conformando las multitudes inteligentes de las que habla Rheingold (2004), que participan en lo que ahora se entiende como activismo móvil. Claras evidencias de ello son el movimiento de los monjes budistas por la democratización en Myanmar y las protestas masivas en Egipto que determinaron la renuncia de Hosni Mubarak tras casi 30 años de gobierno.

Naturalmente, consideramos a estas funciones como mutuamente interdependientes, tal como lo intenta expresar claramente el gráfico 3.

\footnotetext{
1 Personas que se dedican de manera informal y ambulatoria a la compra y venta de divisas, especialmente dólares, en diversos lugares
} de las ciudades del Perú. 


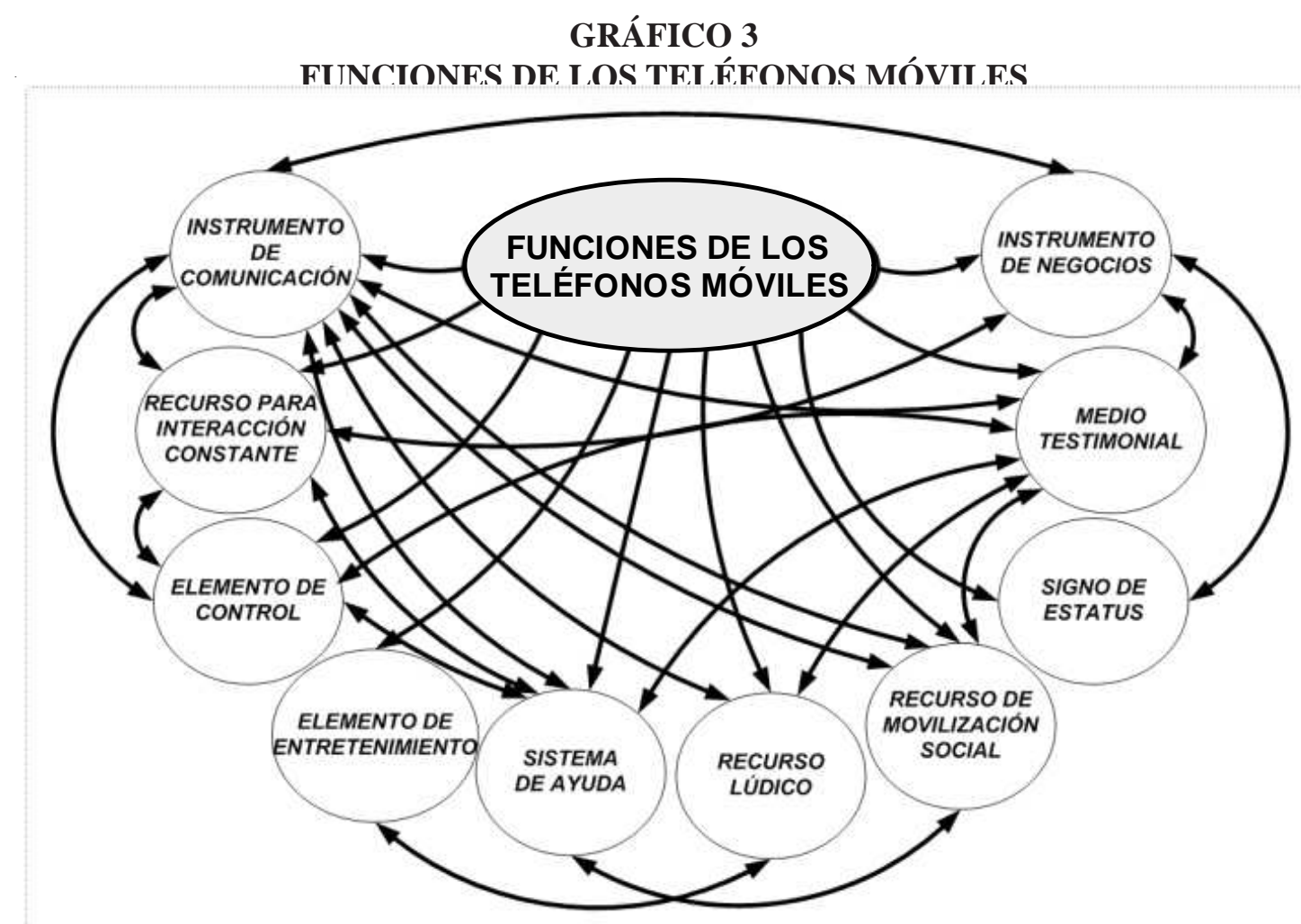

Elaboración: ALR

Finalmente, nos parece bastante útil revisar la perspectiva de van Biljon (2006), quien diferencia cuatro clases de contexto de uso de los teléfonos móviles:

1. Contexto físico: Es el escenario en el cual se desarrolla la comunicación y que por la propia naturaleza del intercambio se moviliza a la par que sus usuarios. Habría que diferenciar las características físicas del aparato como las propias del entorno inmediato que rodea a cada individuo que participa en el intercambio. Puede variar según el autor en cuanto a ubicación. Iluminación, ruido ambiental, temperatura; tanto más las variaciones pueden ser continuas debido al uso del equipo en entornos diversos.

2. Contexto social: Es la interacción social que involucra el empleo del equipo, haciendo posible el intercambio en cualquier momento y en cualquier lugar dentro del rango de servicios del operador. Cualquier usuario se encuentra simultáneamente en dos espacios: El que ocupa físicamente y el espacio virtual de la conversación.

3. Contexto tecnológico: Comprende los recursos del equipo y las redes a las cuales tiene acceso, lo cual depende por supuesto de las condiciones del contrato con el proveedor de servicios.

4. Contexto mental: Involucra la comprensión de los recursos que posee el móvil que se emplea para el intercambio. En el uso del teléfono móvil están implicados recursos cognitivos y metacognitivos. Probablemente el nativo digital accederá a un mayor número de recursos que el migrante digital. No obstante es posible que por las propias peculiaridades de su estilo de uso del móvil, cualquier usuario desconozca o no emplee parte de los recursos con los que cuenta. 


\section{REFERENCIAS}

Andrade, L. (2008) Los SMS: nuevas formas de interacción Juvenil Tesis, Programa de Comunicación, Facultad Latinoamericana de Ciencias Sociales, Quito

Caronia, L. \& Caron, A. (2004) Constructing a Specific Culture: Young People's Use of the Mobile Phone as a Social Performance Convergence: The International Journal of Research into New Media Technologies, 10(2), 28-61

Cassidy, S. (2006) Using social identity to explore the link between a decline in adolescent smoking and an increase in mobile phone use. Health Education, 106, 238-250

Chen-chih C (2005) Study on Mobile Phone Consumption Behavior of College Students and Relational Influential Factors - Take Tainan County and Tainan City As Examples

Faulkner, X. y Culwin, F. (2005) When fingers do the talking: a study of text messaging, Interacting with computers, 17, 167-185

Gergen, K. J. (2002) The challenge of absent presence, In Katz, J. Aakhus, M. (Eds.) Perpetual Contact: Mobile Communication, Private Talk, Public Performance, Cambridge: Cambridge University Press pp. 227-241

Geser, H. (2004) Towards a Sociological Theory of the Mobile Phone Zurich: Sociological Institute. University of Zurich. http://socio.ch/mobile/ $\underline{t \text { geser1.pdf }}$

Гладарев, Б. (2006) Трансформация условий и структур повседневной коммуникации пользователей информационных технологий: на примере мобильной телефонии Диссертация, Институт социологии Российской академии наук, СанктПетербург

Gladarev, B (2006) La transformación de las condiciones y estructuras de la comunicación diaria de los usuarios de tecnología de la información: el caso de la telefonía móvil. Tesis, Instituto de Sociología de la Academia de Ciencias de Rusia, San Petersburgo

Grinter, R. and M. Eldridge. (2001): 'y do tngrs luv 2 txt msg?', in W. Prinz, M. Jarke, Y. Rogers, K.
Schmidt and V. Wulf (eds.): Proceedings of the Seventh European Conference on Computer-Supported Cooperative Work ECSCW '01, Bonn, Germany. Dordrecht, Netherlands: Kluwer Academic Publishers, pp. 219-238.

Grinter, R. and Eldridge, M. 2003. Wan2tlk?: everyday text messaging. In Proceedings of the SIGCHI Conference on Human Factors in Computing Systems (Ft. Lauderdale, Florida, USA, April 05 - 10, 2003). CHI '03. ACM, New York, NY, 441-448.

Humphreys, L. (2010) Mobile social networks and urban public space New Media \& Society, 12(5), 763-778

Langer, J., Moyer, T. \& Stoner, M. (2009) Current Applications of Media Niche Theory in Relationship to College Students Communicating in Local and Long Distant Relationships, Online research paper. http://www.jennalanger.com/wpcontent/uploads/2010/09/LangerJenna-CMC-local-longdistance.pdf

La Rosa, A. (2008) La gente y su aparato: Historias de vida sobre teléfonos celulares. Lima: Instituto Crecimiento

Lasén. A. (2006) La presencia y uso de los teléfonos móviles en los espacios públicos urbanos, Formas de Arquitectura y Arte, 15, 42- 49

Ling, R. (2008) New Tech, New Ties: How Mobile Communication is reshaping social cohesion. Cambridge: MIT

Martínez, I, Aguado, J. (2006) El desarrollo de la tecnología móvil como plataforma mediática Hologramática, Año III, Número 5 (VI), 21-39

Martínez, J. (2008) El hombre posmoderno: Un enfoque humanístico. http://www.lulu.com/items/ volume $67 / 2637000 / 2637087 / 16 /$ print/ 2637087.pdf

Oksman, V. \& Turtiainen, J. (2004) Mobile Communication as a Social Stage: Meanings of Mobile Communication in Everyday Life among Teenagers in Finland New Media \& Society, 6(3), 319339

Palen, L. (2002) Mobile telephony in a connected life March 2002/45(3), COMMUNICATIONS OF THE ACM, 78-82 
Palen, L. y Hughes, A. (2007) When home base is not a place: parents' use of mobile telephones, Personal Ubiquit Computer, 11, 339-348

Prieto, D. (1994) La Vida Cotidiana fuente de Producción Radiofónica Quito: UNDA-AL, 1994

Rheingold, H. (2004) Multitudes inteligentes: La próxima revolución social (Smart Mobs) Barcelona: Gedisa Ediciones

Rettie, R (2009) Mobile phone communication: Extending Goffman to mediated interaction Sociology, 43 (3), 421-438.

Rice, R.E., \& Katz, J. (2003). Mobile discourtesy: National survey results on episodes of convergent public and private spheres. In Nyiri, K. (Eds), Mobile democracy: Essays on society, self and politics. Vienna: Passagen Verlag, pp. 53-64

Satchell, C. (2006) A young nomad's guide to new digital terrain PhD Thesis, School of Applied Communication, RMIT University, Australia

Segui, J. (2009) Nativos digitales y teléfonos móviles: ¿una nueva cultura? Apuntes para una teorización sobre la Sociedad de la Relación y los adolescentes. Ponencia presentada en el XI Congreso Nacional de Psicología Social, Tarragona. Recuperado el 1-3 octubre en: http:// uab.academia.edu/JosepSegui/Papers/267627/
Nativos_digitales_y_telefonos_moviles_una_nueva_cultura _Apuntes_para_una_teorizacion_sobre_la_ Sociedad_de_la_Relacion_y_los_adolescentes

The World Bank (2011) he Little Data Book on Information and Communication Technology 2011, Washington D.C. : The World Bank

van Biljon, J. (2006) A model for representing the motivational and cultural factors that influence mobile phone usage variety $\mathrm{PhD}$ Thesis, University of South Africa

van Biljon, J. \& Krotzké, P. (2008) Cultural factors in a Mobile Phone Adoption and Usage Model Journal of Universal Computer Science, 14(16), 26502679

Walsh, S. (2009) A psychosocial approach to understanding young Australians' mobile phone behaviour, Ph D thesis, School of Psychology and Counseling, Queensland University of Technology, Brisbane, Australia

Walsh, S., White, K., Cox, S. \& Young, R. (2011) Keeping in constant touch: the predictors of young Australians' mobile phone involvement. Computers in Human Behavior, 27(1), 333-342

Watson, A. (2011) The mobile phone: the new communication drum of Papua New Guinea. PhD thesis, Queensland University of Technology.

Fecha de recepción: 20 de noviembre, 2011

Fecha de aceptación: 30 de noviembre, 2011 